\title{
A dosimetric comparison of volumetric modulated arc therapy with three-dimensional conformal radiotherapy in the treatment of cervical cancer
}

\begin{tabular}{|c|c|}
\hline \multicolumn{2}{|c|}{$\begin{array}{l}\text { Authors: } \\
\text { Visham Bhagaloo } \\
\text { Nazreen Bhim }^{1} \text { (D) } \\
\text { Alistair Hunter } \\
\text { Nazia Fakie }^{1} \text { (D) }\end{array}$} \\
\hline \multicolumn{2}{|c|}{$\begin{array}{l}\text { Affiliations: } \\
{ }^{1} \text { Division of Radiation } \\
\text { Medicine, Faculty of Health } \\
\text { Sciences, Groote Schuur } \\
\text { Hospital, University of } \\
\text { Cape Town, Cape Town, } \\
\text { South Africa }\end{array}$} \\
\hline \multicolumn{2}{|c|}{$\begin{array}{l}\text { Corresponding author: } \\
\text { Visham Bhagaloo, } \\
\text { vishambhagaloo@gmail.com }\end{array}$} \\
\hline \multicolumn{2}{|c|}{$\begin{array}{l}\text { Dates: } \\
\text { Received: } 05 \text { Aug. } 2020 \\
\text { Accepted: } 08 \text { Nov. } 2020 \\
\text { Published: } 22 \text { Jan. } 2021\end{array}$} \\
\hline \multicolumn{2}{|c|}{$\begin{array}{l}\text { How to cite this article: } \\
\text { Bhagaloo V, Bhim N, Hunter } \\
\text { A, Fakie N. A dosimetric } \\
\text { comparison of volumetric } \\
\text { modulated arc therapy with } \\
\text { three-dimensional conformal } \\
\text { radiotherapy in the } \\
\text { treatment of cervical cancer. } \\
\text { S. Afr. j. oncol. 2021;5(0), } \\
\text { a149. https://doi. } \\
\text { org/10.4102/sajo.v5i0.149 }\end{array}$} \\
\hline \multicolumn{2}{|c|}{$\begin{array}{l}\text { Copyright: } \\
\text { (C) 2021. The Authors } \\
\text { Licensee: AOSIS. This } \\
\text { is licensed under the } \\
\text { Creative Commons } \\
\text { Attribution License. }\end{array}$} \\
\hline \multicolumn{2}{|l|}{ Read online: } \\
\hline 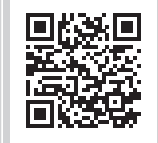 & $\begin{array}{l}\text { Scan this QR } \\
\text { code with your } \\
\text { smart phone or } \\
\text { mobile device } \\
\text { to read online. }\end{array}$ \\
\hline
\end{tabular}

Background: External beam radiotherapy (EBRT) is essential in the management of locally advanced cervical cancer (LACC). Volumetric modulated arc therapy (VMAT) is thought to achieve higher conformity to the planned target volume (PTV) and better sparing of organs at risk (OAR) when compared to three-dimensional conformal radiotherapy (3D-CRT). This study focused on these principles as it applied to treatment and potential toxicity in the management of LACC.

Aim: The aim of this study was to compare dosimetric parameters between VMAT and 3D-CRT in the management of LACC.

Setting: The study analysed patients treated at Groote Schuur Hospital (GSH) between May and December 2017.

Methods: This is a non-randomised comparative retrospective study. Three-dimensional conformal radiation and VMAT plans were generated, and data on treatment parameters for PTV D50\%, Dmax, Dmean, conformity index (CI), homogeneity index (HI), treated volume (TV), irradiated volume (IV) and OAR constraints; femoral heads, bladder, bowel bag, rectum and bone marrow were collected.

Results: Of the 45 patients assessed, VMAT showed statistically significant, lower treatment parameter values for CI (1.09 vs. 1.49) and TV $\left(1613.1 \mathrm{~cm}^{3}\right.$ vs. $\left.2230.3 \mathrm{~cm}^{3}\right)$, whereas 3D-CRT showed lower Dmax (48.1 Gy vs. $49.2 \mathrm{~Gy})$ and IV (10652.2 $\mathrm{cm}^{3}$ vs. $\left.14618.1 \mathrm{~cm}^{3}\right)$. Volumetric modulated arc therapy OAR doses revealed a lower V45 for bowel bag $\left(182.3 \mathrm{~cm}^{3} \mathrm{vs}\right.$. $\left.411.3 \mathrm{~cm}^{3} ; p<0.001\right)$, lower V40 for bone marrow $(19.1 \%$ vs. $38.7 \% ; p<0.001)$ and rectum $(88.5 \%$ vs. $96 \% ; p<0.001)$. A reduced 3D-CRT dose was noted for bladder Dmax (47.4 Gy vs. $48.3 \mathrm{~Gy} ; p<0.001)$.

Conclusion: Volumetric modulated arc therapy offered a superior dosimetric option, with better OAR dose sparing and optimal tumour dosimetry.

Keywords: cervical cancer; VMAT; 3D-CRT; radiation therapy; dosimetry.

\section{Introduction}

Globally, cervical cancer is the fourth most frequent cancer in women, representing 6.6\% (57 000 cases) in 2018, of which $90 \%$ of deaths occurred in low- and middle-income countries. ${ }^{1}$ In 2014 , South Africa registered 5735 new cases of cervical cancer, representing $15.17 \%$ of all female cancers, and the third most common cancer in women. ${ }^{2}$ In South Africa, there is limited data regarding the stage of presentation; however, at Groote Schuur Hospital (GSH), a higher percentage of patients treated for cervical cancer present with locally advanced disease. Locally advanced cervical cancer (LACC) (Stage 1B2 - IVA [International Federation of Gynaecology and Obstetrics (FIGO) 2009]) is associated with a poorer prognosis with 5-year survival rates of $58 \%$ in Stage IIB, 30\% in Stage III and 16\% in Stage IVA. ${ }^{3}$ Cervical cancer continues to affect middle-aged females and has been associated with numerous socio-economic factors, especially in lowermiddle-income countries. ${ }^{4}$ Considering these factors, appropriate, beneficial, minimally toxic and proven efficacious treatment is imperative.

Locally advanced cervical cancer is managed definitively with external beam radiotherapy (EBRT) and concurrent chemotherapy followed by brachytherapy. ${ }^{5}$ External beam radiotherapy is indicated in approximately $60 \%$ of patients with cervical cancer, treated with curative intent ${ }^{6}$ as surgical intervention is limited to early-stage disease, mainly Stage 1A and 1B1. Three-dimensional 
conformal radiation therapy (3D-CRT) is the most widely used method of EBRT $^{7}$ but has been associated with significant toxicity including genito-urinary symptoms, gastrointestinal symptoms and bone marrow suppression, particularly when combined with concurrent chemotherapy. ${ }^{8}$ At GSH, all LACC patients treated radically with curative intent to receive concurrent chemotherapy with radiotherapy, increasing possible toxicities. It is with this concern in mind that volumetric modulated arc therapy (VMAT) was considered to improve dosimetric optimisation and potentially minimise toxicity for patients treated with EBRT for LACC.

Volumetric modulated arc therapy is a form of intensitymodulated radiation therapy (IMRT) linking together variable dose rates and gantry speeds, a single gantry motion and dynamic multi-leaf collimation. ${ }^{9}$ Patients with cervical cancer who were treated with IMRT have reported improved target volume coverage, which also allowed for dose escalation whilst reducing the radiation dose to organ at risk (OAR). ${ }^{10}$ Additional studies had shown contradictory information suggesting no dosimetric or clinical benefit with selected OAR sparing when IMRT was used. ${ }^{11,12}$ The ability to offer improved coverage whilst sparing OAR may allow for a superior therapeutic effect of VMAT when compared to 3D-CRT. Taking this into consideration and the contrasting published results, this study may help to determine the better dosimetric option at our institution.

The incidence and severity of radiation toxicity are multifactorial. These factors include the volume of tissue treated, dose received $\left(\mathrm{D}_{2 \%}\right.$ and $\left.\mathrm{D}_{\text {mean }}\right)$ and the technique for delivery of radiation therapy (3D-CRT vs. IMRT) administered. Radiation Therapy Oncology Group (RTOG) 1203 compared IMRT with 3D-CRT radiotherapy in cervical cancer. The results showed significantly lower scores for gastrointestinal and urinary toxicity when using IMRT. ${ }^{13}$ The study utilised the Expanded Prostate Cancer Index Composite (EPIC) to compare toxicities. Expanded Prostate Cancer Index Composite is a questionnaire used to assess the function and toxicity post-treatment. The mean EPIC bowel score declined 23.6 points in the 3D-CRT group and 18.6 points in the IMRT group. The mean urinary score declined 10.4 points in the standard radiotherapy group and 5.6 points in the IMRT group. A larger decline from baseline suggested a poorer tolerance to treatment. Radiation Therapy Oncology Group 418 further suggested that IMRT was associated with lower rates of haematological toxicities with a lower $\mathrm{V}_{40}$ volume and higher rates of cisplatin use when compared to 3D-CRT. ${ }^{14}$

With the advent of VMAT, issues regarding target definition, patient and target immobilisation, tissue deformation and reproducibility remain to be validated as additional factors that limit the OAR toxicity and treatment profiles. ${ }^{12,15,16}$ To minimise the effects listed, specialised contouring guidelines for cervical cancer VMAT treatments have been published and implemented. ${ }^{15}$ Additionally, with improved imaging localisation such as daily kilovoltage cone-beam computed tomography (CT) as compared to megavoltage orthogonal images, tumour localisation, deformation and reproducibility may be minimised. ${ }^{17}$

Studies comparing conventional techniques to VMAT in the management of LACC are scarce and patient populations are small. ${ }^{9}$ To date, there are no published articles in South Africa comparing 3D-CRT with VMAT. At GSH, VMAT, the accepted standard of treatment internationally, ${ }^{7,8,9}$ was added as a treatment option in May 2017. This study aims to provide clarity on the dosimetrically superior option between these two modalities.

\section{Methods Study aims and objectives}

The aim of this study was to determine if VMAT is dosimetrically superior to 3D-CRT in the treatment of patients with LACC staged with the 2009 International Federation of Gynaecology and Obstetrics (FIGO) system. The study included patients who were treated with VMAT at GSH between May and December 2017.

\section{Study population}

Formal sample size calculations were not performed. The number of patients was based on the feasibility of data collection and processing. The patients included were those treated radically for LACC with VMAT and concurrent chemotherapy, had a minimum age of 18 years and an Eastern Cooperative Oncology Group (ECOG) performance status of $0-2$. Patients not meeting these criteria were excluded from the study. Further exclusion criteria were connective tissue disease, poor performance status, previous pelvic radiotherapy, previous surgery and metastatic disease on imaging.

\section{Scientific design}

\section{Methods and materials}

This is a non-randomised comparative retrospective study. The medical records of all patients treated for LACC between May and December 2017 meeting the inclusion criteria were reviewed. Data relating to age, stage and histological type were recorded. The patients' VMAT radiation therapy plans were reviewed and the following information extracted: PTV $\mathrm{D}_{50 \%}, \mathrm{D}_{\text {max }}, \mathrm{D}_{\text {mean }}$, conformity index $(\mathrm{CI})$, homogeneity index (HI), treated volume (TV), irradiated volume (IV) and OAR constraints; right and left femoral heads, bladder, bowel bag, rectum and bone marrow. For this study, appropriate 3D-CRT plans were generated and used solely for dosimetric comparison.

\section{Treatment}

All patients were discussed at the combined gynaecology clinic before treatment commenced. These patients were prescribed a bowel prep with Senna/Docusate and lactulose for 2 weeks prior to CT scan simulation and continued throughout treatment to aid with rectal emptying and reproducibility. 
On the day of treatment, a bladder protocol was followed: patients were asked to empty their bladders and then drink 250 cubic centimetres (cc) of water, followed by a 30-min waiting period before CT simulation. This bladder protocol was repeated daily whilst on treatment to further improve bladder reproducibility. At GSH an inferior tumour limit is determined clinically, and the corresponding CT location marked to aid with lower limit delineation of tumours as magnetic resonance imaging is not routinely available for all patients. Patients were aligned using the triangulation technique and scanned with intravenous and oral contrast in the supine position with knee rest and ankle stocks using 5 millimetres $(\mathrm{mm})$ slices from T12 vertebral body to the mid femur. On the scan date, tattooing of the skin with threepoint pelvic markings was performed to improve the setup and minimise rotational errors during treatment. The final CT images were imported and contoured using the Eclipse planning system.

The contouring guidelines ${ }^{15,17,18}$ utilised at GSH generated a clinical target volume (CTV). The CTV tumour comprised of the gross tumour volume (GTV), cervix, uterus, parametrium and vagina. Gross Tumour Volume delineation without MRI imaging was exceptionally difficult. As a result, it was assumed that once the cervix was contoured the disease was encompassed. Vaginal contouring was dependent on the extent of the disease. For minimal or no vaginal involvement, the upper half of the vagina was contoured. If the upper half of the vagina was involved with tumour, the upper two-thirds were contoured and for extensive disease the entire vagina was contoured. Additionally, the mesorectum was included for Stage III B or extensive nodal involvement. The CTV nodes were generated using a 0.7 centimetres $(\mathrm{cm})$ margin around the common, internal and external iliac vessels whilst using a $1.0 \mathrm{~cm}$ strip anterior to the sacrum. The obturator nodes were contoured with a $1.0 \mathrm{~cm}$ strip, which connected the external and internal iliac vessels. Inguinal nodes with a $0.7 \mathrm{~cm}$ expansion were contoured when there were round ligament and lower third of the vaginal involvement. The final planning target volume (PTV) included the PTV tumour, created with a $1.5 \mathrm{~cm}$ expansion superior, anterior and posterior with $1.0 \mathrm{~cm}$ in all other directions from the CTV, and the PTV node, generated with a symmetric $0.7 \mathrm{~cm}$ expansion circumferentially from the CTV. The OARs - right and left femoral head, bladder, bowel bag, bone marrow ${ }^{19}$ and rectum - were also delineated using RTOG guidelines for the female pelvis. ${ }^{18}$ The patient's final plans were prescribed to a dose of 46.0 Gray (Gy) to the mean volume in 23 fractions.

External beam radiotherapy plans were generated using the Eclipse system and optimisation criteria for VMAT technique. The plans were evaluated by the attending radiation oncologist based on the following criteria: PTV coverage (volume covered by the $95 \%$ isodose line), dose maximum (highest doses within the PTV which should be less than $107 \%$ for $\mathrm{D}_{\max }$ and $\mathrm{D}_{2 \%}$ ), hot spots (a significant dose and corresponding volume (typically $<2$ cubic centimetres $\left[\mathrm{cm}^{3}\right]$ and $>107 \%$ dose) outside of the PTV), monitor units (MU) (suggested as less than $300 \mathrm{MU}$ per Gy for VMAT), HI, CI and OAR constraints as defined by Quantec. ${ }^{20}$ The plans were approved once all criteria were assessed and found to be within acceptable limits. The approved plans were then assessed by the Medical Physics Department using quality assurance protocols. The gamma index was then evaluated before the plan was finally approved and treatment began. At $\mathrm{GSH}$, a difference of $3 \%$ dose and a $3 \mathrm{~mm}$ geometrical location between the planned and calculated doses on the treatment machines are accepted. All patients with LACC treated radically also received concurrent weekly cisplatin at a dose of 40 milligrams per square metres $\left(\mathrm{mg} / \mathrm{m}^{2}\right)$. Chemotherapy should ideally be commenced on Mondays to ensure a synergistic effect with chemotherapy and radiotherapy. However, because of logistical issues, chemotherapy is given on a Thursday at GSH. Daily setup and verification was confirmed using offline electronic portal imaging device (EPID) verification. Additionally, brachytherapy commenced within the last week of EBRT using a two-dimensional (2D) system prescribed to a Manchester point system.

The patients selected for this study, who met the inclusion criteria, had a second plan generated using 3D-CRT. Senior radiographers with experience in 3D-CRT cervical cancer were used and blinded to previous radiotherapy plans with VMAT to reduce any possible bias. The plans were generated with mix energy beams, 18 Mega-voltage (MV) and 6 MV photon energy, and no restrictions were placed on the number of fields or use of beam modifiers. At GSH, a four-field box with varying beam energies was typically used for pelvic PTV structures to ensure appropriate coverage and minimal toxicity to OAR. The final assessment and approval followed the criteria previously mentioned. Nine (20\%) of the 3D-CRT plans were rejected as the criteria were not met. These included poor superior PTV coverage, OAR exceeded constraint and unacceptable hot spots. The plans were accepted after suggested corrections were made. All 45 plans were again reviewed by an independent oncologist and, once agreed, forwarded for quality assurance. External plan validation was also completed with the IMSureTM system, Standard Imaging which utilises an independent planning system to verify that the dose prescribed was the dose given.

\section{Dosimetric data}

Post plan assessment recorded PTV $\mathrm{D}_{2 \%}$, PTV D ${ }_{98 \%}$, PTV D $\mathrm{D}_{50 \% \text {, }}$ $\mathrm{D}_{\text {max' }} \mathrm{D}_{\text {mean }}, \mathrm{CI}, \mathrm{HI}, \mathrm{TV}, \mathrm{IV}$ and patient separation to fully assess treatment parameters.

TV: The volume enclosed by an isodose surface, selected by the radiation oncologist to achieve the purpose of treatment. ${ }^{21}$ At GSH the 95\% isodose was used.

IV: The volume of tissue that receives a dose that is considered significant in relation to normal tissue tolerance. ${ }^{21}$ At GSH the $20 \%$ isodose was used.

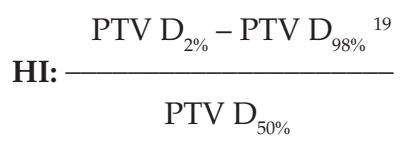




\section{CI: \\ PTV Volume}

Patients' OAR doses or volumes were recorded based on Quantec; right and left femoral head maximum dose $\left(\mathrm{D}_{\max }<\right.$ $52 \mathrm{~Gy}){ }^{22}$ bladder maximum dose $\left(\mathrm{D}_{\max }<65 \mathrm{~Gy}\right){ }^{22}$ small bowel (bowel bag) absolute volume $\left(\mathrm{V}_{45}<195 \mathrm{cc}\right)$, $^{22}$ bone marrow relative volume $\left(\mathrm{V}_{40}<40 \%\right)^{23}$ and rectum relative volume $\left(\mathrm{V}_{40}<100 \%\right)^{13}$ were assessed.

\section{Statistical consideration}

Statistical analysis was performed using SPSS Version 25. Comparison of dosimetric values for 3D-CRT to VMAT was performed using Wilcoxon signed rank test as the initial Shapiro-Wilk test showed that the data were not normally distributed. Additionally, Mann-Whitney $U$ tests were used when comparing cervical cancer by stage. A $p<0.05$ was deemed statistically significant.

\section{Ethical considerations}

Throughout the study, patient privacy and confidentiality were strictly maintained. Anonymity was upheld by de-identifying the patients; the data collected were stored on REDCap. Ethical approval for the study was obtained from the Human Research Ethics Committee, Faculty of Health Sciences, University of Cape Town (Reference Number HREC REF: 625/2018).

\section{Results}

\section{Patient characteristics}

The mean age of the 45 patients was $52.8 \pm 10.8$ years.

The most common stage of disease identified was Stage IIB $(n=22)$ followed by Stage IIIB $(n=15)$. The other stages are illustrated in Figure 1.

The most common histology was squamous cell carcinoma $(n=38)$ as shown in Figure 1, with adenocarcinomas representing seven patients.

\section{Tumour dosimetry}

Table 1 examines the dosimetric values recorded for both treatment planning options. The median PTV volume for the patients treated was $1450 \mathrm{~cm}^{3}$ and a $\mathrm{D}_{\max }$ value favouring 3D-CRT. Additionally, PTV $\mathrm{D}_{2 \%}$ was reviewed, which is considered a volume assigned to a maximum dose rather than a point dose, and no statistical difference was found between 3D-CRT and VMAT.

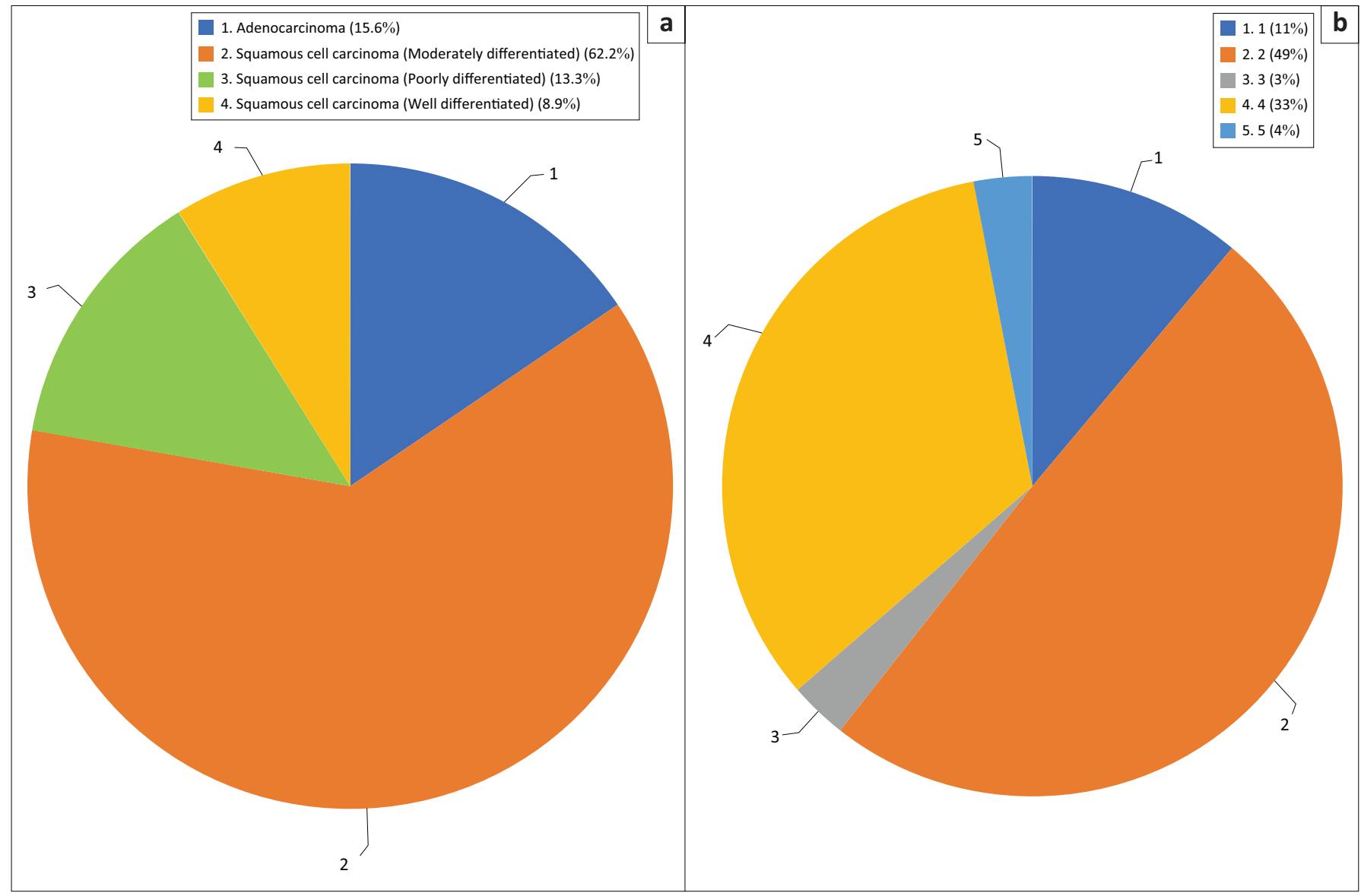

FIGURE 1: (a) Percentage distribution by histological sub-classification type. (b) Percentage distribution by stages of cervical cancer disease. 
TABLE 1: Tumour dosimetric parameters for volumetric modulated arc therapy and three-dimensional conformal radiation.

\begin{tabular}{|c|c|c|c|c|c|c|}
\hline \multirow[t]{2}{*}{ Variables } & \multicolumn{2}{|c|}{ VMAT } & \multicolumn{2}{|l|}{ 3D-CRT } & \multirow[t]{2}{*}{$z$} & \multirow[t]{2}{*}{$p$} \\
\hline & Median & IQR & Median & IQR & & \\
\hline PTV $D_{2 \%}$ (Gy) & 47.8 & $47.5-48.0$ & 47.7 & $47.4-48.3$ & -0.70 & 0.483 \\
\hline PTV D ${ }_{98 \%}(G y)$ & 44.1 & $43.8-44.4$ & 44.4 & $44.2-44.6$ & -2.97 & 0.003 \\
\hline PTV D ${ }_{50 \%}$ (Gy) & 46.0 & $46.0-46.1$ & 46.6 & $46.1-46.9$ & -5.45 & $<0.001$ \\
\hline Homogeneity index & 0.08 & $0.07-0.09$ & 0.07 & $0.06-0.08$ & -1.47 & 0.142 \\
\hline$D_{\max }(G y)$ & 49.2 & $48.9-49.5$ & 48.1 & $47.7-48.6$ & -5.37 & $<0.001$ \\
\hline$D_{\text {mean }}(G y)$ & 46.0 & $46.0-46.0$ & 46.5 & $46.1-46.7$ & -5.17 & $<0.001$ \\
\hline Treated volume $\left(\mathrm{cm}^{3}\right)$ & 1613.1 & $1471.4-1776.4$ & 2230.3 & $1960.3-2457.9$ & -5.84 & $<0.001$ \\
\hline PTV volume $\left(\mathrm{cm}^{3}\right)$ & 1443.9 & $1331.8-1661.2$ & 1443.9 & $1331.8-1661.2$ & -0.37 & 0.715 \\
\hline Conformity index & 1.09 & $1.06-1.12$ & 1.49 & $1.44-1.59$ & -5.84 & $<0.001$ \\
\hline Irradiated volume $\left(\mathrm{cm}^{3}\right)$ & 14618.1 & $12844-17943.2$ & 11910.4 & $10652.2-14272.9$ & -5.77 & $<0.001$ \\
\hline
\end{tabular}

VMAT, volumetric modulated arc therapy; 3D-CRT, 3D conformal radiation; IQR, Interquartile range; PTV, planned target volume; Gy, Gray.

TABLE 2: Volumetric modulated arc therapy versus three-dimensional conformal radiation organ at risk dosimetry.

\begin{tabular}{lccccccc}
\hline Organ & \multicolumn{2}{c}{ VMAT } & & \multicolumn{2}{c}{ 3D-CRT } & $p$ \\
\cline { 2 - 3 } & Median & IQR & & Median & IQR & \\
\hline Right femoral head (Gy) & 45.6 & $44.8-46.8$ & & 46.6 & $46.1-47.3$ & $<0.001$ \\
Left femoral head (Gy) & 45.4 & $44.0-46.2$ & & 46.8 & $45.7-47.4$ & $<0.001$ \\
Bladder (Gy) & 48.3 & $47.9-48.7$ & & 47.4 & $47.0-47.9$ & $<0.001$ \\
Bowel bag (cm ${ }^{3}$ ) & 182.3 & $129.3-250.2$ & & 411.3 & $342.1-541.4$ & $<0.001$ \\
Bone marrow (Vol \%) & 19.1 & $14.9-24.9$ & & 38.7 & $33.9-43.2$ & $<0.001$ \\
Rectum (Vol \%) & 88.5 & $83.3-96.0$ & & 96.0 & $90.0-99.8$ & $<0.001$ \\
\hline
\end{tabular}

VMAT, volumetric modulated arc therapy; 3D-CRT, 3D conformal radiation; IQR, Interquartile range; Gy, Gray.

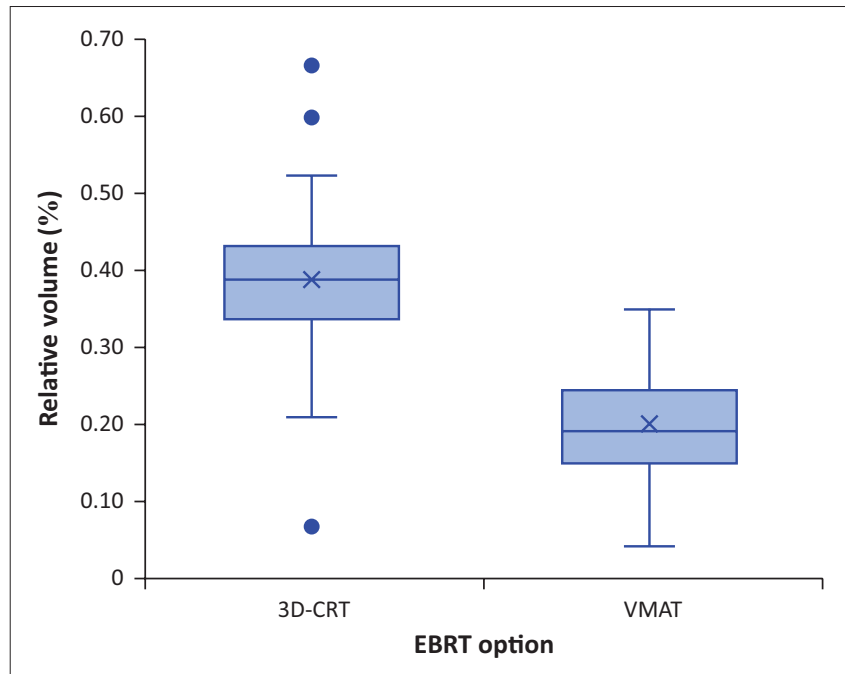

EBRT, external beam radiotherapy; VMAT, volumetric modulated arc therapy; 3D-CRT, 3D conformal radiation.

FIGURE 2: Box and whisker plot comparing bone marrow relative volume at $40 \mathrm{~Gy}$.

The CI for VMAT showed a lower median value when compared to 3D-CRT whilst the improved HI associated with 3D-CRT was deemed statistically insignificant. Dosimetric assessment also showed approximately a $600 \mathrm{~cm}^{3}$ smaller median TV and a $2700 \mathrm{~cm}^{3}$ larger IV for VMAT when compared to 3D-CRT.

\section{Organs at risk}

Volumetric modulated arc therapy displayed lower median maximum doses when compared to 3D-CRT for both femoral heads (Table 2). Figure 2 compares the relative

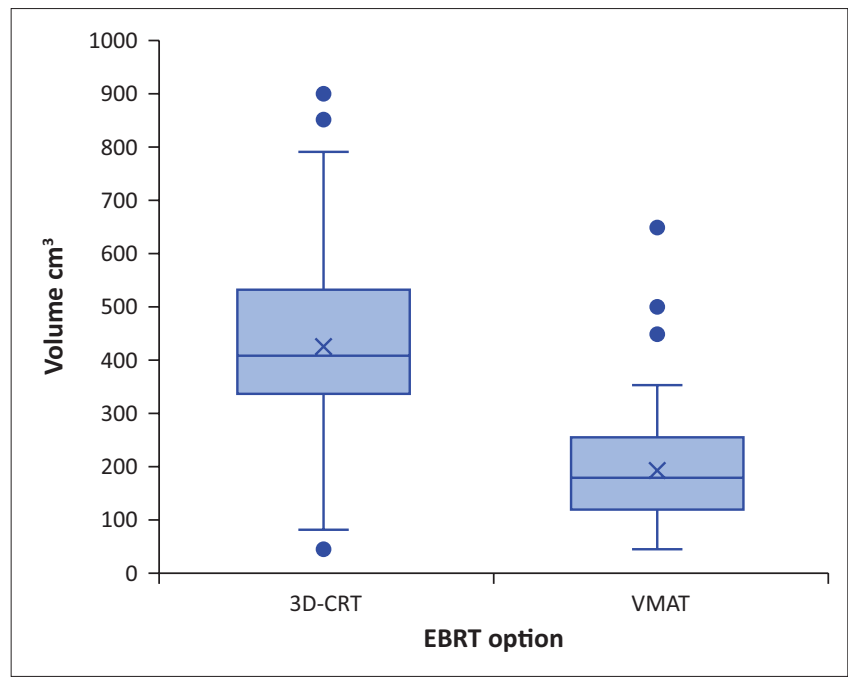

EBRT, external beam radiotherapy; VMAT, volumetric modulated arc therapy; 3D-CRT, 3D conformal radiation.

FIGURE 3: Box and whisker plot comparing bowel bag absolute volume at 45 Gy.

volume of bone marrow receiving $40 \mathrm{~Gy}$, showing a two times larger volume affected with 3D-CRT as compared to VMAT. Figure 3 compares the volume of bowel receiving a dose of $45 \mathrm{~Gy}$ and shows a median volume of $200 \mathrm{~cm}^{3}$ greater with 3D-CRT than that recorded for VMAT. Median bladder $\mathrm{D}_{\max }$ was lower for 3D-CRT when compared to VMAT (Table 2). All OAR dosimetric values are presented in Table 2.

Rectum, $\mathrm{V}_{50}<50$ as per Quantec was not used as dose maximum in all plans was less than 50.0 Gy, making dosimetric comparisons impossible, as such $\mathrm{V}_{40}<100 \%$ was used. ${ }^{13}$ The results showed a significant reduction in relative volume received, favouring VMAT.

\section{Sub-group analysis}

When comparing Stage IIB with Stage IIIB cancer groups, statistically significant differences were only found for VMAT TV95 Isodose $(U=83), 3 \mathrm{D}-\mathrm{CRT}$ TV 95 Isodose $(U=70)$ and PTV $(U=83)$. As per data in Table 3, no difference was noted for OAR dosimetry. 
TABLE 3: Volume comparisons between three-dimensional conformal radiation and volumetric modulated arc therapy for Stages IIB and IIIB.

\begin{tabular}{|c|c|c|c|c|c|}
\hline \multirow[t]{2}{*}{ Variables } & \multicolumn{2}{|r|}{ Stage IIB } & \multicolumn{2}{|r|}{ Stage IIIB } & \multirow[t]{2}{*}{$p$} \\
\hline & Median & IQR & Median & IQR & \\
\hline $\begin{array}{l}\text { VMAT treated } \\
\text { volume }\end{array}$ & 1558.5 & $1461.23-1693.83$ & 1754.70 & $1571.50-1993.30$ & 0.01 \\
\hline $\begin{array}{l}3 \mathrm{D} \text { treated } \\
\text { volume }\end{array}$ & 2188.6 & $1940.5-2340.03$ & 2473.00 & 2309.90-2616.70 & 0.003 \\
\hline PTV volume & 1429.10 & $1295.10-1579.25$ & 1658.10 & $1439.2-1862.7$ & 0.01 \\
\hline
\end{tabular}

VMAT, volumetric modulated arc therapy; 3D-CRT, 3D conformal radiation; PTV, planned target volume.

\section{Discussion}

The aim of this study focused on determining the dosimetrically superior EBRT option in the management of LACC at GSH, South Africa. To date, no similar studies have been carried out in South Africa comparing 3D-CRT with VMAT in the management of LACC. Most institutions with the capability to offer IMRT have carried out so with little local evidence or dosimetric proof of superiority. Threedimensional conformal radiation therapy remains the preferred treatment reference, to which all inverse planning options are compared when managing LACC. ${ }^{23,24}$ This study examined dosimetric values for OAR and assessed tumour dosimetry between 3D-CRT and VMAT to determine the superior option.

Both 3D-CRT and VMAT showed appropriate dose coverage of the PTV. This coverage was deemed appropriate once a minimum of the $95 \%$ isodose line encompassed the PTV. Additionally, the PTV $\mathrm{D}_{\text {max }}$ and $\mathrm{D}_{\text {mean }}$ were found to be within an acceptable range as outlined in the International Commission on Radiation Units and Measurements (ICRU) guidelines. ${ }^{25}$ Furthermore, quantitative analysis of the plans showed VMAT having a superior, statistically significant CI compared to 3D-CRT (Table 1). Similar results were presented in the study carried out by Guy et al. in 2016 comparing the dosimetrically superior option in LACC. ${ }^{9}$ Conformity index values closer to 1 suggest a more conformal distribution of higher doses as represented by TV. As radiotherapy moves towards greater precision, improved conformity is essential in tumour dose escalation ${ }^{26}$ and minimising high dose exposure to OAR.

One of the major goals of radiotherapy is to deliver the maximum prescribed dose to a selected target volume uniformly, thus increasing the likelihood of tumour control. The HI, defined as the uniformity of dose distribution in the target volume, gives a numerical representation of this distribution. The HI between both methods of treatment was numerically different, indicating a small benefit towards 3D-CRT (Table 1). However, the value was not deemed statistically significant. At GSH, an HI less than 0.1 is accepted, which was achieved in both EBRT options.

It has been suggested that IMRT offers superior OAR sparing in gynaecological malignancies. ${ }^{27}$ Similar findings in this study have supported this. Organ at risk toxicity may be affected by maximum dose as seen with the right femoral head, left femoral head and bladder, whilst volumedependent doses may affect organs such as bowel bag, rectum and bone marrow. Small bowel toxicity, as determined for bowel bag contours, suggests a $<10 \%$ Grade 3 toxicity for late effects and acute effects when V45 Gy $<195$ $\mathrm{cm}^{3} \cdot{ }^{15}$ The 3D-CRT median dose from this study revealed a value of $411.3 \mathrm{~cm}^{3}$ receiving $45 \mathrm{~Gy}$, whilst $182.3 \mathrm{~cm}^{3}$ for VMAT (Table 2 and Figure 3). Mell et al. suggested a correlation between this dosimetric value and clinical symptoms tending to less acute and chronic symptoms when smaller volumes are exposed to similar doses. ${ }^{28}$ Rectal dose comparisons also showed a more favourable dosimetric value for VMAT and as seen in RTOG 1203, a possible decrease in clinical toxicity. ${ }^{29}$

Bone marrow contouring guidelines and dose constraints ${ }^{30}$ have been well documented in cervical cancer patients. Clinical toxicity, secondary to bone marrow suppression, with EBRT appears to have a greater clinical impact when combined with concurrent chemotherapy as opposed to EBRT alone. ${ }^{6}$ At GSH, this was noted in previous observational studies which altered the timely delivery of EBRT, especially in patients receiving concurrent chemotherapy. In the study population, the 3D-CRT patients' median absolute volume receiving 40 Gy was twice that of VMAT (Table 2 and Figure 2 ), considerably increasing the risk of Grade 2 and greater bone marrow toxicity. Bone marrow toxicity secondary to EBRT may result in delayed treatments which are in turn associated with poorer outcomes. In a study carried out by Girinsky et al. the results showed when treatment exceeded 52 days, loss of local control and overall survival was approximately $1 \%$ per day. ${ }^{31}$

Whilst VMAT was superior in limiting OAR dose constraints to most organs contoured, 3D-CRT offered a reduced maximum dose to the bladder (Table 2). This result is contradictory to similar studies including Lin et al., whose research documented lower bladder doses in a meta-analysis of cervical cancer treatment with IMRT. ${ }^{32}$ It has been postulated that limited prioritisation for the bladder contoured in the optimisation software used by the staff members at GSH may be the reason for the difference in values. Both VMAT and 3D-CRT bladder max doses were within Quantec ${ }^{15}$ constraints and it was difficult to determine the extent of the clinical impact of these differences.

Volumetric modulated arc therapy can reduce the treated volume (TV) by improving the CI. It also limits the OAR toxicity by allowing multiple-beam entry points as it rotates around a patient, avoiding OAR contours. This sparing effect has led to an increase in lower doses from multiple small fields. The IV represents that volume of tissue irradiated by a clinically significant dose. This study revealed a significantly larger IV with VMAT when compared to 3D-CRT (Table 1). Concern regarding secondary malignancies with increased integral dose as represented by IV has been studied extensively and most studies suggest that integral dose is not a good estimator of quantifying cancer induction. ${ }^{33}$ 
Lastly, sub-group analysis (Table 3) also revealed no statistically significant increase in OAR dosimetric values in Stage IIB versus Stage IIIB cervical cancer when treated with either 3D-CRT or VMAT. The significant volume difference is based on unique guidelines used for Stage IIIB, which includes contouring of the mesorectum and vagina depending on the degree of involvement. These data suggest that whilst Stage IIIB results in larger contoured PTV tumour volumes, the OAR dosimetric values were comparable to Stage IIB.

\section{Limitations}

This study has its limitations, including the retrospective nature and small sample size. Additionally, the dosimetric data gathered represent numerical values, which were compared against Quantec ${ }^{15}$ guidelines to determine the possibility of clinical complications. These guidelines, whilst not extensively validated for VMAT, have been the backbone to which dosimetric values are compared to minimise clinical OAR toxicity.

\section{Conclusion}

Minimising toxicities whilst optimising tumour treatment is essential in the successful management of LACC. The data analysed for the investigated study population showed the dosimetric benefit of VMAT over 3D-CRT in the management of LACC, with improved OAR sparing. Improved OAR sparing with lower doses to the femoral heads, bowel bag, rectum and bone marrow, as seen with VMAT planning, will possibly reduce both early and late toxicities. Volumetric modulated arc therapy also offered comparable tumour coverage and dosimetry, equally homogenous dose distribution, with the added benefits of improved conformity and possible dose escalation. Maintaining and improving tumour dosimetry with a reduction in toxicity allows for better compliance with treatment, which is integral in improving outcomes. The authors are optimistic that this study will provide support towards VMAT as a treatment option for LACC at GSH whilst remaining vigilant of new uncertainties.

\section{Acknowledgements}

The authors would like to thank Prof. J. Parkes and all staff at GSH and UCT who were instrumental in this publication. Special mention to the Radiotherapists (RTT) Team at Groote Schuur Hospital who created the 3D-CRT plans for this study.

\section{Competing interests}

The authors have declared that no competing interest exists.

\section{Authors' contributions}

Data collection, analysis and manuscript composition were carried out by V.B. Review, expert consultation and final documentation approval were carried out by N.F., A.H. and N.B.

\section{Funding information}

This research received no specific grant from any funding agency in the public, commercial or not-for-profit sectors.

\section{Data availability statement}

Data sharing is not applicable to this article as no new data were created or analysed in this study.

\section{Disclaimer}

The views and opinions expressed in this article are those of the authors and do not necessarily reflect the official policy or position of any affiliated agency of the authors.

\section{References}

1. World Health Organization. Cervical cancer [homepage on the Internet]. 2018 [cited 2020 Jan 19]. Available from: https://www.who.int/cancer/prevention/ diagnosis-screening/cervical-cancer/en/

2. National Institute for communicable disease (NIFC). Cancer in South Africa 2014 full report [document on the Internet]. National Cancer Registry South Africa; 2014 [cited 2019 Jan 19]. Available from: https://www.nicd.ac.za/wp-content/ uploads/2019/12/2014-NCR-tables.pdf

3. Society AC. Prognosis in locally advanced cervical cancer [homepage on the Internet]. 2015 [updated 2020 Jan 3; cited 2020 Jan 19]. Available from: https:// www.cancer.org/cancer/cervical-cancer/detection-diagnosis-staging/survival.html

4. Denny L, Anorlu R. Cervical cancer in Africa. Cancer Epidemiol Biomarkers Prev. 2012;21(9):1434-1438. https://doi.org/10.1158/1055-9965.EPI-12-0334

5. Koh WJ, Abu-Rustum NR, Bean S, et al. Cervical cancer, version 3.2019, NCCN clinical practice guidelines in oncology. J Natl Compr Canc Netw. 2019;17(1):64-84. https://doi.org/10.6004/jnccn.2019.0001

6. Delaney G, Jacob S, Barton M. Estimation of an optimal radiotherapy utilization rate for gynecologic carcinoma. Cancer. 2004;101(4):671-681. https://doi. org/10.1002/cncr.20445

7. Peters WA, III, Liu PY, Barrett RJ, II, et al. Concurrent chemotherapy and pelvic radiation therapy compared with pelvic radiation therapy alone as adjuvant therapy after radical surgery in high-risk early-stage cancer of the cervix. J Clin Oncol. 2000;18(8):1606-1613. https://doi.org/10.1200/JCO.2000.18.8.1606

8. Samper-Ternent R, Zhang D, Kuo Y-F, Hatch S, Freeman J. Late GI and bladder toxicities after radiation for uterine cancer. Gynecol Oncol. 2011;120(2):198-204. https://doi.org/10.1016/j.ygyno.2010.10.017

9. Guy JB, Falk AT, Auberdiac $P$, et al. Dosimetric study of volumetric arc modulation with RapidArc and intensity-modulated radiotherapy in patients with cervical cancer and comparison with 3-dimensional conformal technique for definitive cancer and comparison with 3-dimensional conformal technique for definitive
radiotherapy in patients with cervical cancer. Med Dosim. 2016;41(1):9-14 radiotherapy in patients with cervical cancer.
https://doi.org/10.1016/j.meddos.2015.06.002

10. Chen M-F, Tseng C-J, Tseng C-C, Kuo Y-C, Yu C-Y, Chen W-C. Clinical outcome in posthysterectomy cervical cancer patients treated with concurrent cisplatin and intensity-modulated pelvic radiotherapy: Comparison with conventional radiotherapy. Int J Radiat Oncol Biol Phys. 2007;67(5):1438-1444. https://doi. org/10.1016/j.ijrobp.2006.11.005

11. Yang B, Zhu L, Cheng H, Li Q, Zhang Y, Zhao Y. Dosimetric comparison of intensity modulated radiotherapy and three-dimensional conformal radiotherapy in patients with gynecologic malignancies: A systematic review and meta-analysis. Radiat Oncol. 2012;7:197. https://doi.org/10.1186/1748-717X-7-197

12. Erpolat OP, Alco G, Caglar HB, et al. Comparison of hematologic toxicity between 3DCRT and IMRT planning in cervical cancer patients after concurrent chemoradiotherapy: A national multi-center study. Eur J Gynaecol Oncol. 2014;35(1):62-66.

13. Klopp AH, Yeung AR, Deshmukh S, et al. Patient-reported toxicity during pelvic intensity-modulated radiation therapy: NRG Oncology-RTOG 1203. J Clin Oncol. 2018;36(24):2538-2544. https://doi.org/10.1200/JCO.2017.77.4273

14. Klopp AH, Moughan J, Portelance L, et al. Hematologic toxicity in RTOG 0418: A phase 2 study of postoperative IMRT for gynecologic cancer. Int J Radiat Oncol Biol Phys. 2013;86(1):83-90. https://doi.org/10.1016/j.jirobp.2013.01.017

15. Lim K, Small Jr, W, Portelance L, et al. Consensus guidelines for delineation of clinical target volume for intensity-modulated pelvic radiotherapy for the definitive treatment of cervix cancer. Int J Radiat Oncol Biol Phys. 2011;79(2):348-355. https://doi.org/10.1016/j.ijrobp.2009.10.075

16. Loiselle $C$, Koh WJ. The emerging use of IMRT for treatment of cervical cancer. J Natl Compr Canc Netw. 2010;8(12):1425-1434. https://doi.org/10.6004/ jnccn. 2010.0106

17. Taylor A, Rockall AG, Reznek RH, Powell MEB. Mapping pelvic lymph nodes: Guidelines for delineation in intensity-modulated radiotherapy Int J Radiat Oncol Biol Phys. 2005;63(5):1604-1612. https://doi.org/10.1016/j. ijrobp.2005.05.062 
18. RTOG. Female RTOG normal pelvis [homepage on the Internet]. 2020 [cited 2020 Jan 20]. Available from: https://www.nrgoncology-org/Portals/0/Scientific\%20 Program/CIRO/Atlases/Female\%20RTOG\%20Normal\%20Pelvis\%20Atlas.pdf

19. Report 83. J ICRU. 2016;10(1):NP. https://doi.org/10.1093/jicru/10.1.Report83

20. Bentzen SM, Constine LS, Deasy JO, et al. Quantitative analyses of normal tissue effects in the clinic (QUANTEC): An introduction to the scientific issues. Int J Radiat Oncol Biol Phys. 2010;76(3):S3-S9. https://doi.org/10.1016/j.jijobp.2009.09.040

21. Landberg T, Chavaudra J, Dobbs J, et al. Report 50. J ICRU. 2016;os26(1):NP. https://doi.org/10.1093/jicru/os26.1.Report50

22. Marks LB, Yorke ED, Jackson A, et al. Use of normal tissue complication probability models in the clinic. Int J Radiat Oncol Biol Phys. 2010;76(Suppl 3):S10-S19. https://doi.org/10.1016/j.ijrobp.2009.07.1754

23. Morris M, Eifel PJ, Lu J, et al. Pelvic radiation with concurrent chemotherapy compared with pelvic and para-aortic radiation for high-risk cervical cancer. N Engl Med. 1999;340(15):1137-1143. https://doi.org/10.1056/NEJM199904153401501

24. Randriamanovontsoa E, Ratsimandresy D, Rakotonarivo J, Rakototiana AF, Rantomalala H, Rafaramino F. Result of concurrent chemoradiotherapy for cervical cancer at radiation oncology service of university hospital Joseph Ravoahangy Andrianavalona from 2007 to 2009. Pan Afr Med J. 2014;19:298. https://doi. org/10.11604/pamj.2014.19.298.4350

25. Ahmad R, Hoogeman MS, Quint S, Mens JW, Osorio EMV, Heijmen BJM. Residual setup errors caused by rotation and non-rigid motion in prone-treated 2012;103(3):322-326. https://doi.org/10.1016/j.radonc.2012.04.013

26. Kam MK, Chau RM, Suen J, Choi PH, Teo PM. Intensity-modulated radiotherapy in nasopharyngeal carcinoma: Dosimetric advantage over conventional plans and feasibility of dose escalation. Int J Radiat Oncol Biol Phys. 2003;56(1):145-157. https://doi.org/10.1016/S0360-3016(03)00075-0
27. Roeske JC, Lujan A, Rotmensch J, Waggoner SE, Yamada D, Mundt AJ. Intensitymodulated whole pelvic radiation therapy in patients with gynecologic modulated whole pelvic radiation therapy in patients with gynecologic
malignancies. Int J Radiat Oncol Biol Phys. 2000;48(5):1613-1621. https://doi. org/10.1016/S0360-3016(00)00771-9

28. Mell LK, Sirák I, Wei L, et al. Bone marrow-sparing intensity modulated radiation therapy with concurrent cisplatin for stage IB-IVA cervical cancer: An international multicenter phase II clinical trial (INTERTECC-2). Int J Radiat Oncol Biol Phys. 2017;97(3):536-545. https://doi.org/10.1016/j.ijrobp.2016.11.027

29. Yeung AR, Pugh SL, Klopp AH, et al. Improvement in patient-reported outcomes with intensity-modulated radiotherapy (RT) compared with standard RT: A report from the NRG oncology RTOG 1203 study. J Clin Oncol. 2020;38(15):1685-1692. https://doi.org/10.1200/JCO.19.02381

30. Mahantshetty U, Krishnatry R, Chaudhari S, et al. Comparison of 2 contouring methods of bone marrow on CT and correlation with hematological toxicities in non-bone marrow-sparing pelvic intensity-modulated radiotherapy with concurrent cisplatin for cervical cancer. Int J Gynecol Cancer. 2012;22(8): 1427-1434. https://doi.org/10.1097/IGC.0b013e3182664b46

31. Girinsky T, Rey A, Roche B, et al. Overall treatment time in advanced cervical carcinomas: A critical parameter in treatment outcome. Int J Radiat Oncol Biol Phys. 1993;27(5):1051-1056. https://doi.org/10.1016/03603016(93)90522-W

32. Lin Y, Chen K, Lu Z, et al. Intensity-modulated radiation therapy for definitive treatment of cervical cancer: A meta-analysis. Radiat Oncol. 2018;13(1):177. https://doi.org/10.1186/s13014-018-1126-7

33. D’Arienzo M, Masciullo SG, De Sanctis V, Osti MF, Chiacchiararelli L, Enrici RM Integral dose and radiation-induced secondary malignancies: Comparison between stereotactic body radiation therapy and three-dimensional conforma radiotherapy. Int J Environ Res Public Health. 2012;9(11):4223-4240. https://doi. org/10.3390/ijerph9114223 